\title{
Composición y preferencia de materiales en nidos de vertebrados nidificadores de cavidades en el bosque templado andino de Chile
}

\author{
Composition and preferences regarding nest materials by cavity-nesting \\ vertebrates in the Andean temperate forest of Chile
}

\author{
María Teresa Honorato a, Tomás A Altamirano a*, José Tomás Ibarra ${ }^{\text {a,b,c }}$, \\ Mariano De la Maza ${ }^{\text {a,d }}$, Cristián Bonacic a, Kathy Martin ${ }^{\text {c,e }}$ \\ *Autor de correspondencia: ${ }^{a}$ Pontificia Universidad Católica de Chile, Facultad de Agronomía e Ingeniería Forestal, \\ Departamento de Ecosistemas y Medio Ambiente, Laboratorio Fauna Australis, Santiago, Chile, taaltami@uc.cl \\ b Pontificia Universidad Católica de Chile, Centro de Desarrollo Local (CEDEL), Campus Villarrica, Villarrica, Chile. \\ c University of British Columbia, Department of Forest and Conservation Sciences, \\ Centre for Applied Conservation Research, Vancouver, BC, Canada.

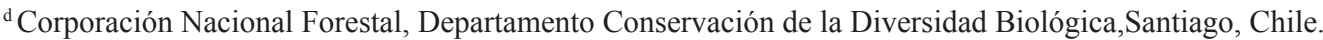 \\ ${ }^{\mathrm{e}}$ Environment Canada, Pacific Wildlife Research Centre, Delta, BC, Canada.
}

\begin{abstract}
SUMMARY
The composition and quality of nesting materials may influence the breeding success of cavity-nesting vertebrates, providing protection and optimal temperature to eggs and chicks. Between 2010 and 2013, we studied the composition and preferences regarding nest materials used by cavity-nesters in Andean temperate forests, southern Chile. We deployed 240 nest-boxes, obtaining data for 290 nests for three bird species (Aphrastura spinicauda, Troglodytes aedon and Tachycineta meyeni) and four mammals (Dromiciops gliroides, Rattus rattus, Oligoryzomys longicaudatus and Irenomys tarsalis). Aphrastura spinicauda $(\mathrm{n}=170)$ and D. gliroides $(\mathrm{n}=91)$ comprised $90 \%$ of the nests. A. spinicauda nests were composed mainly of vine tendrils $(44.2 \%)$ and branches of trees and shrubs (25.7\%). Dromiciops gliroides used mostly leaves of trees and shrubs $(72.9 \%)$, and bryophytes $(20.2 \%)$. The predominant and preferred leaf in the nests of both species was from bamboo Chusquea species. Aphrastura spinicauda avoided the leaves of Lophozonia obliqua, whereas $D$. gliroides avoided vines. These results suggest a previously unknown role of Chusquea spp. in the breeding ecology of cavity-nesting forest vertebrates. The thick matted nests and the acidic $\mathrm{pH}$ of the Chusquea spp. leaves may provide protection from predators and have a biocidal effect on pathogens. Chusquea spp. may have an important influence on the breeding success of cavity-nesters, although the benefits of using this nest material need to be confirmed.
\end{abstract}

Key words: Aphrastura spinicauda, bamboo, breeding, Dromiciops gliroides, resource selection.

\section{RESUMEN}

La composición y calidad de los nidos pueden afectar el éxito reproductivo de vertebrados nidificadores de cavidades, brindándoles protección y temperatura adecuada a huevos, polluelos y crías. Entre 2010-2013, se estudió la composición y preferencia de materiales de nidos de vertebrados nidificadores de cavidades del bosque templado andino de Chile. Se instalaron 240 cajas-nidos, obteniendo 290 nidos de tres especies de aves (Aphrastura spinicauda, Troglodytes aedon y Tachycineta meyeni) y de cuatro mamíferos (Dromiciops gliroides, Rattus rattus, Oligoryzomys longicaudatus e Irenomys tarsalis). El $90 \%$ de los nidos correspondieron a $A$. spinicauda $(\mathrm{n}=170)$ y $D$. gliroides $(\mathrm{n}=91)$. Los nidos de $A$. spinicauda estuvieron compuestos principalmente por zarcillas de trepadoras $(44,2 \%)$ y ramas de árboles y arbustos $(25,7 \%)$. Los nidos de $D$. gliroides estuvieron compuestos principalmente por hojas (72,9\%) y briófitas $(22,9 \%)$. Dentro del ítem hojas, la hoja predominante y preferida por ambas especies fue de Chusquea spp. Aphrastura spinicauda rechazó las hojas de Lophozonia obliqua. Dromiciops gliroides rechazó las zarcillas de trepadoras. Estos resultados sugieren un rol relevante de Chusquea spp. en la ecología reproductiva de las especies estudiadas. Los enmarañados y grandes nidos de Chusquea spp., junto con el pH ácido de sus hojas, podrían proporcionar tanto una protección contra depredadores, como un efecto biocida contra patógenos. Chusquea spp. podría influenciar el éxito reproductivo de vertebrados nidificadores de cavidades, pero su beneficio debe ser dilucidado.

Palabras clave: Aphrastura spinicauda, bambú, Dromiciops gliroides, reproducción, selección de recursos. 


\section{INTRODUCCIÓN}

La construcción de nidos para la reproducción, hibernación y/o descanso, es una actividad muy común en aves, mamíferos, reptiles e insectos (Hansell 2000). Entre los vertebrados de bosque, numerosas especies en el mundo requieren cavidades en árboles para reproducirse (Martin y Eadie 1999). En general, estas especies construyen nidos en el interior de cavidades para obtener protección y temperatura adecuada para sus huevos, polluelos y crías (Collias y Collias 1984). Los materiales utilizados en los nidos podrían ser determinantes en el éxito reproductivo de los nidificadores de cavidades por el papel que ejercen en la selección sexual, protección contra potenciales depredadores, junto con la mantención de una temperatura y un $\mathrm{pH}$ adecuados (Hilton et al. 2004, Álvarez y Barba 2009, Mainwaring et al. 2014). Por ejemplo, distintos materiales regulan la tasa de enfriamiento de los huevos de forma diferencial, donde las plumas serían el mejor aislante y el pasto el peor, revirtiéndose este indicador cuando los materiales están mojados (Hilton et al. 2004). Así, Tachycineta meyeni Cabanis (golondrina chilena), en la etapa de construcción de su nido, agrega menos plumas a medida que la temperatura diaria media aumenta (Liljesthröm et al. 2009). Las hojas son consideradas materiales semi-aislantes, pero estas mantienen su propiedad aun cuando se encuentran mojadas (Reid et al. 2002). Por otra parte, algunos materiales verdes (e.g., hojas, zarcillas de trepadoras, briófitas) pueden contener compuestos secundarios volátiles y un $\mathrm{pH}$ que evita la proliferación de patógenos (Clark y Mason 1985, Dubiec et al. 2013). A pesar del importante rol que podrían cumplir los distintos materiales utilizados en la construcción de nidos por especies nidificadoras de cavidades, el conocimiento detallado sobre la composición de estos es principalmente anecdótico (exceptuando Liljesthröm et al. 2009).

La selección (i.e., proceso que implica decisiones conductuales hechas por el animal en cuanto a qué recurso utilizar) podría mostrar diferencias con la preferencia (i.e., utilización desproporcionada de un recurso al compararlo con lo esperado por su disponibilidad en el ambiente) de materiales utilizados en los nidos de las especies nidificadoras de cavidades (Hall et al. 1997). La distinción entre selección y preferencia es importante, ya que la utilización de ciertos materiales podría reflejar el proceso de selección asociado pero, en algunos casos, los recursos utilizados podrían no corresponder con la disponibilidad de materiales en los hábitats de las especies (Hall et al. 1997). Por ejemplo, Troglodytes aedon Vieillot (chercán) se reproduce ampliamente en hábitats con distintos grados de perturbación, pudiendo reemplazar los materiales del nido por los que encuentra disponibles en su hábitat modificado (Atienzar et al. 2010). Así, esta ave selecciona una gran variedad de materiales para nidificar, sin embargo, no se ha evaluado cuantitativamente si es que esta especie muestra alguna preferencia por alguno de estos.
Un $57 \%$ de la comunidad de aves (29 especies) y al menos cinco mamíferos utilizan cavidades para reproducirse en el bosque templado sudamericano (Altamirano 2014). Sin embargo, aún es escaso el conocimiento sobre la reproducción de los vertebrados nidificadores de cavidades en el bosque templado de Chile y Argentina. En estos ecosistemas se han realizado estudios sobre pequeños paseriformes utilizando cajas-nidos, los que han evaluado principalmente la ecología reproductiva de dos especies de aves: T. aedon y Aphrastura spinicauda Gmelin (rayadito) (e.g. Moreno et al. 2005, Ippi et al. 2012, Altamirano et al. 2015). Sólo dos de estos estudios describen de manera general la composición de nidos de A. spinicauda. En Chiloé, los nidos de esta especie estuvieron compuestos por pequeñas ramas, rizomas, finos tallos de helechos, hojas de Chusquea spp. (bambú, colihue o quila), herbáceas y pelos (Moreno et al. 2005). En la región del Maule, la composición de los nidos de $A$. spinicauda se basó principalmente en acículas de Pinus radiata D. Don. (pino insigne), ramas de árboles y arbustos, junto con plumas, musgos, epífitas, herbáceas y pelos (Quilodrán et al. 2012). Para los mamíferos, Celis-Diez et al. (2012) describieron que el nido de Dromiciops gliroides Thomas (monito del monte) estaba compuesto tanto de epífitas, como de trepadoras, briófitas y vegetación de sotobosque como hojas de Chusquea spp. en la isla de Chiloé (sur de Chile). Jiménez y Rageot (1979) describieron 22 nidos de D. gliroides encontrados en la zona continental, los que en la mayoría de los casos fueron construidos de hojas de Chusquea spp. Sin embargo, también se encontraron nidos hechos completamente de hojas secas de Lapageria rosea Ruiz et Pav. (copihue), o de musgos y líquenes. La amplia variabilidad de materiales descritos en los nidos de A. spinicauda y D. gliroides, los que podrían tener funciones diferentes en las distintas partes del nido, sugeriría que ambas especies no prefieren ningún material en particular para la construcción de estos. Sin embargo, ninguno de estos estudios evaluó en detalle la composición de los nidos utilizados para reproducirse por ambas especies. Menos aún ha sido evaluada la relación entre los materiales utilizados en los nidos y su disponibilidad en el hábitat, lo que informaría sobre la preferencia o rechazo de ciertos materiales bajo distintas condiciones de hábitat.

En este trabajo se evalúa la composición y preferencia/ rechazo de materiales en nidos de pequeños vertebrados que utilizan cavidades en el bosque templado andino del sur de Chile. Específicamente, (i) se describe la composición de nidos de tres especies de aves y cuatro mamíferos, y (ii) se evalúa si dos especies (A. spinicauda y $D$. gliroides) prefieren o rechazan algún material en relación a lo disponible en el hábitat reproductivo. El primer objetivo es de naturaleza descriptiva. Para el segundo objetivo se predice que $A$. spinicauda y $D$. gliroides no mostrarán preferencia ni rechazo por algún material específico. Para desarrollar estos objetivos se instalaron cajas-nido, con el supuesto de que la composición de los nidos en ca- 
vidades naturales no varía respecto a los construidos en cajas-nido, lo que ha sido visualmente observado en estudios paralelos con cavidades naturales (Altamirano 2014).

\section{MÉTODOS}

Especies y área de estudio. Se estudiaron los nidos de especies que se reproducen y/o hibernan al interior de cavidades de árboles. Se determinó la especie a la cual correspondía cada nido por observación directa de los adultos (monitoreo regular cada 2-3 días de cada nido) y/o observación mediante cámaras trampas (ver detalles en Altamirano et al. 2015). Los nidos de micromamíferos corresponden a periodos reproductivos e hibernación. De esta manera, las especies estudiadas corresponden a las aves A. spinicauda, T. aedon y T. meyeni, y los mamíferos D. gliroides, Rattus rattus Linnaeus (rata negra), Irenomys tarsalis Philippi (rata arbórea) y Oligoryzomys longicaudatus Bennett (ratón de cola larga).

Los bosques del centro-sur y sur de Chile se encuentran dentro de la influencia bioclimática templada, la cual abarca desde el paralelo $35^{\circ}$ hasta $55^{\circ}$ de Sudamérica. El presente estudio se desarrolló en la zona andina de la comuna de Pucón, región de La Araucanía ( $\left.39^{\circ} \mathrm{S}\right)$, Chile. En zonas bajas (200-900 m s.n.m.), dominan especies arbóreas de los géneros Nothofagus, Lophozonia, Persea, Gevuina, Laurelia, Laureliopsis, y Saxegothaea. En zonas altas (> 900 m s.n.m.) dominan los bosques antiguos mixtos de Nothofagus pumilio Poepp. et Endl. Krasser (lenga) con Araucaria araucana Molina. K. Koch. (araucaria) (Rojas et al. 2011). En el sotobosque es común la presencia de especies del género Chusquea, numerosos helechos y arbustos. Las plantas trepadoras más comunes en el área de estudio son Boquila trifoliolata DC. Decne. (voqui blanco), Cissus striata Ruiz et Pav. (voqui colorado), L. rosea, Hydrangea serratifolia Hook. et Arn. F.Phil. (voqui paulún) y Mitraria coccinea Cav. (botellita).

Colecta y tratamiento de nidos. En septiembre de 2010 se instalaron 240 cajas-nido en seis sitios. En cada sitio se instalaron 40 cajas-nido, manteniéndolas disponibles para su uso durante tres temporadas reproductivas (20102013). Las cajas-nido fueron distribuidas uniformemente en grillas de $8 \times 5$ cajas-nido, separadas por una distancia mínima de $25 \mathrm{~m}$ entre ellas. Estas cajas-nido se instalaron colgando de ramas que se encontraban entre 1,5 y $2,5 \mathrm{~m}$ de altura (Altamirano et al. 2015). Las cajas-nido tuvieron una entrada de diámetro $3,1 \mathrm{~cm}$, y sus medidas internas eran de $16,5 \mathrm{~cm}$ de largo, 13,2 $\mathrm{cm}$ de ancho y $17,1 \mathrm{~cm}$ de profundidad desde la base de la entrada. Una vez finalizada cada temporada reproductiva, se colectaron los nidos para su análisis en laboratorio.

La estructura de los nidos fue descrita en relación a las siguientes variables: peso (se utilizó una balanza digital de precisión $0,1 \mathrm{a} 1.100 \mathrm{~g}) \mathrm{y}$ volumen $\left(\mathrm{cm}^{3}\right.$, alto $\mathrm{x}$ largo $\mathrm{x}$ ancho); para el caso de las aves se obtuvo además, la profun- didad, largo y ancho de la taza interna (Hansell 2000). En el caso de las aves, para obtener el volumen total se restó el volumen de la taza interna. Para determinar la composición de los nidos, estos fueron secados en una estufa de convección a $105^{\circ} \mathrm{C}$ por cinco horas. Luego, los nidos se disgregaron y los materiales de cada nido fueron clasificados en los siguientes ítems: plumas, pelos, briófitas (principalmente musgos y hepáticas), líquenes, hojas, ramas de árboles y arbustos (en adelante ramas), zarcillas de trepadoras (en adelante trepadoras), semillas, corteza, chips de madera, barro, tela de araña y materiales no naturales (e.g., nylon, plástico e hilo) (Atienzar et al. 2010). Se determinó la presencia o ausencia de cada material descrito dentro de cada nido y se calculó la proporción que ocupaban dentro del nido, obteniendo la frecuencia de ocurrencia para cada ítem analizado. Los ítems hojas, ramas y semillas se lograron identificar a nivel de especie, estimando visualmente la presencia y proporción del volumen total de éstas en cada nido.

Evaluación del hábitat reproductivo e hibernación. Una vez terminada la temporada reproductiva, se determinó la estructura y composición de la vegetación circundante a cada caja-nido. Se realizaron parcelas circulares de 0,04 ha (11,2 m de radio) con cada caja-nido como centro (Altamirano 2014). Dentro de cada parcela, se registraron las siguientes variables: cobertura de sotobosque general, sotobosque de Chusquea spp. y la composición de especies leñosas dentro de la parcela. Para cada árbol con diámetro a la altura del pecho (DAP) mayor a $12,5 \mathrm{~cm}$, se registró la especie, el DAP y la cobertura de trepadoras en el tronco y copa (la cual fue estimada utilizando las siguientes categorías: $0: 0 \%$ cobertura; $1: 1-25 \%$; $2: 26-50 \%$; $3: 51-75 \%$ y 4: 76-100 \%) (Altamirano 2014).

Análisis de datos. Ningún individuo fue marcado, por lo tanto no fue posible determinar si los nidos entre temporadas correspondieron a las mismas parejas. Los datos fueron analizados separadamente para cada especie que utilizó las cajas-nido. Sólo en el caso de I. tarsalis y $O$. longicaudatus, la composición de sus nidos se analizó en conjunto debido a la dificultad de diferenciar los nidos de ambas especies cuando no se encontraron adultos en el interior (en adelante "roedor nativo" hará referencia a los nidos de ambas especies, ya que el otro roedor estudiado - R. rattuses una especie exótica en el área). Los siguientes ítems se agruparon en la categoría "otros": líquenes, semillas, corteza, chips de madera, barro, tela de araña y materiales no naturales, debido a la baja presencia de estos en los nidos. Los análisis para comparar los materiales encontrados en los nidos y la disponibilidad de estos recursos en el hábitat reproductivo, fueron realizados sólo para $A$. spinicauda y $D$. gliroides, ya que ambas especies totalizaron el $90 \%$ de los nidos analizados $(\mathrm{n}=261)$. Se realizó un análisis no paramétrico de Spearman para evaluar la correlación entre la proporción de Chusquea spp. y trepadoras encontradas 
en el nido, y lo observado en cada parcela. Por otra parte, se evaluó preferencia/rechazo de materiales utilizados respecto a lo disponible en el hábitat por medio de pruebas de chi cuadrado $\left(\chi^{2}\right)$. Específicamente, para ambas especies se evaluó la preferencia/rechazo de hojas de Chusquea spp., hojas de Lophozonia obliqua Mirb. Heenan et Smissen (roble) y trepadoras. Además, para A. spinicauda se evaluó la preferencia/rechazo de hojas de Nothofagus dombeyi Mirb. Oerst. (coigüe) y acículas de Pinus radiata. Los resultados son reportados de la siguiente manera: $\chi^{2}$ grados de libertad, tamaño de la muestra $=$ valor chi cuadrado; valor $P$. Los análisis fueron realizados utilizando el programa JMP 9®.

\section{RESULTADOS}

Se analizó un total de 290 nidos durante las tres temporadas reproductivas, de los cuales 170 fueron de A. spinicauda, 91 de D. gliroides, 16 de $R$. rattus, nueve de roedor nativo, tres de T. aedon y uno de T. meyeni. Para A. spinicauda, el peso promedio del nido fue de $36,8 \pm$ $11,9 \mathrm{~g}$ (rango $=9,4-69,9 \mathrm{~g})$. El peso promedio del nido de $D$. gliroides fue de 41,2 $\pm 22,6 \mathrm{~g}$ (rango $=6,5-100,2 \mathrm{~g}$ ). Los nidos más livianos entre las seis especies correspondieron a los de $A$. spinicauda, seguido por los de D. gliroides, mientras que los nidos más pesados correspondieron a los de $R$. rattus (cuadro 1). Los nidos menos altos correspondieron a $A$. spinicauda, con un promedio de 3,7 $\pm 0,9 \mathrm{~cm}$ (rango $=1,8-7,8 \mathrm{~cm}$ ), mientras que los nidos más altos correspondieron a los de $R$. rattus con un promedio de 6,9 $\pm 2,8 \mathrm{~cm}$ (rango 3,0 - 11,6 cm). Además, los nidos de $A$. spinicauda tuvieron una base de materiales largos y finos (ramas de árboles y arbustos, y zarcillas de trepadoras), donde construían su taza interna. Luego, ellos revestían esta taza interna con materiales aislantes como pelos y plumas, manteniendo su forma aún fuera de la caja-nido. De igual manera, los nidos de D. gliroides tenían una estructura entretejida, principalmente con hojas de Chusquea spp., donde en ocasiones agregaban briófitas. Por otro lado, los nidos de $R$. rattus no mostraron una estructura entretejida, donde las hojas que agregaban en él solo eran depositadas y acumuladas, perdiendo el nido su estructura fuera de la cavidad.

En cuanto a la composición de los nidos, $A$. spinicauda fue la única especie que presentó, en todas las temporadas reproductivas, todos los ítems analizados (figura 1). Los nidos de $A$. spinicauda estuvieron compuestos mayoritariamente por zarcillas de trepadoras $(44,1 \%)$ y ramas de árboles y arbustos $(28,7 \%)$. Por otra parte, los nidos de D. gliroides estuvieron compuestos principalmente por hojas $(66,9 \%)$ y briófitas $(17,9 \%)$. Todos los mamíferos, junto con $T$. aedon, utilizaron en mayor proporción el ítem hojas (figura 1). Para todas las especies, a excepción de $R$. rattus, la hoja de mayor proporción dentro de los nidos fue de Chusquea spp. (figura 2). La mayor proporción de hojas de Chusquea spp. fue encontrada en los nidos de
T. aedon, con un $91,7 \%$ del total de las hojas. Para todas las especies nativas, a excepción de $T$. aedon, la segunda hoja de mayor proporción correspondió a L. obliqua. Los nidos de A. spinicauda estuvieron compuestos en un 22,6\% de hojas de L. obliqua del total de hojas presentes en sus nidos (figura 2).

Para A. spinicauda se encontró una correlación positiva entre la proporción de Chusquea spp. en el nido y lo disponible en el hábitat reproductivo $\left(P<0,001, \mathrm{r}_{\mathrm{s}}=\right.$ $0,540)$. Sin embargo, no se encontró una correlación entre la proporción de trepadoras en el nido y en el hábitat reproductivo $\left(P=0,240, \mathrm{r}_{\mathrm{s}}=0,090\right)$. Para $D$. gliroides, aumentó la proporción de Chusquea spp. $(P<0,001, \mathrm{r}=0,840)$ y trepadoras $\left(P=0,040, \mathrm{r}_{\mathrm{s}}=0,220\right)$ en el nido al aumentar la proporción de estos ítems en el hábitat reproductivo.

Aphrastura spinicauda no mostró preferencia/rechazo para los ítems trepadoras $(100 \%$ de presencia en parcelas y nidos) y hojas de $N$. dombeyi $\left(\chi_{1,170}^{2}=0,601, P>0,05\right)$, seleccionando estos acorde a lo disponible en el hábitat reproductivo. Sin embargo, $A$. spinicauda seleccionó las hojas de Chusquea spp. $\left(\chi_{1,170}^{2}=29,450, P<0,001\right)$ y las acículas de $P$. radiata $\left(\chi_{1,170}^{2}=10,870, P<0,001\right)$ positiva y desproporcionalmente en relación a lo disponible (i.e., preferencia). Al contrario, a pesar de encontrarse presente en el $72 \%$ de los hábitats reproductivos, la frecuencia de hojas de L. obliqua en los nidos fue menor a la esperada (i.e., rechazo de este material; $\chi_{1,170}^{2}=11,43, P<0,001$ ). Por su parte, D. gliroides mostró una preferencia por Chusquea spp. $\left(\chi_{1,91}^{2}=6,28, P=0,010\right)$ y un rechazo por las trepadoras $\left(\chi_{1,91}^{2}=12,248, P<0,001\right)$.

\section{DISCUSIÓN}

Este estudio entrega una evaluación de la composición y preferencia/rechazo de materiales en nidos de vertebrados nidificadores de cavidades que habitan la parte septentrional del bosque templado andino de Sudamérica. Los resultados indican que las aves y pequeños mamíferos estudiados usan una amplia gama de componentes naturales (plumas, pelos, briófitas, líquenes, hojas, ramas de árboles y arbustos, zarcillas de trepadoras, semillas, corteza, restos de madera, barro, tela de araña) y, en muy baja proporción, materiales no naturales (nylon, plástico e hilo). A pesar de esta diversidad de recursos utilizados, los resultados resaltan la importancia de Chusquea spp. en los nidos de las especies estudiadas. Las hojas de Chusquea spp. fueron las de mayor proporción en todas las especies nativas estudiadas y fue preferida por sobre otros materiales tanto por A. spinicauda como por D. gliroides.

Existieron diferencias entre los componentes utilizados por las especies estudiadas en relación a los pocos antecedentes que existen para estas en otras zonas de su distribución. Estas diferencias sugieren que las especies del bosque templado serían principalmente generalistas en el uso de recursos (Vuilleumier 1985, Ibarra y Martin 2015), adaptándose y modificando los materiales utilizados en sus 
Cuadro 1. Peso ( $\mathrm{g}$ ) y volumen total $\left(\mathrm{cm}^{3}\right)$ de los nidos analizados por especie (media \pm desviación estándar) para Aphrastura spinicauda, Troglodytes aedon, Tachycineta meyeni, Dromiciops gliroides, Rattus rattus, Irenomys tarsalis y Oligoryzomys longicaudatus.

Weight $(\mathrm{g})$ and total volume $\left(\mathrm{cm}^{3}\right)$ of nests (mean \pm standard deviation) for Aphrastura spinicauda, Troglodytes aedon, Tachycineta meyeni, Dromiciops gliroides, Rattus rattus, Irenomys tarsalis and Oligoryzomys longicaudatus.

\begin{tabular}{lcccc}
\hline \multicolumn{1}{c}{ Especie } & $\mathrm{n}$ & Peso $(\mathrm{g})$ & Volumen total $\left(\mathrm{cm}^{3}\right)$ & Temporada \\
\hline Aphrastura spinicauda & 170 & $37 \pm 11,9$ & $657 \pm 0,5$ & $2010-2013$ \\
Troglodytes aedon & 3 & $50 \pm 24,4$ & $1.407 \pm 29,7$ & $2010-2012$ \\
Tachycineta meyeni & 1 & 54 & 926 & $2012-2013$ \\
Dromiciops gliroides & 91 & $41 \pm 22,6$ & $1.325 \pm 12,7$ & $2010-2013$ \\
Rattus rattus & 16 & $64 \pm 35,5$ & $2.200 \pm 27,8$ & $2011-2013$ \\
Irenomys tarsalis/Oligoryzomys longicaudatus & 9 & $56 \pm 20,9$ & $1.496 \pm 7,6$ & $2010-2013$ \\
\hline
\end{tabular}
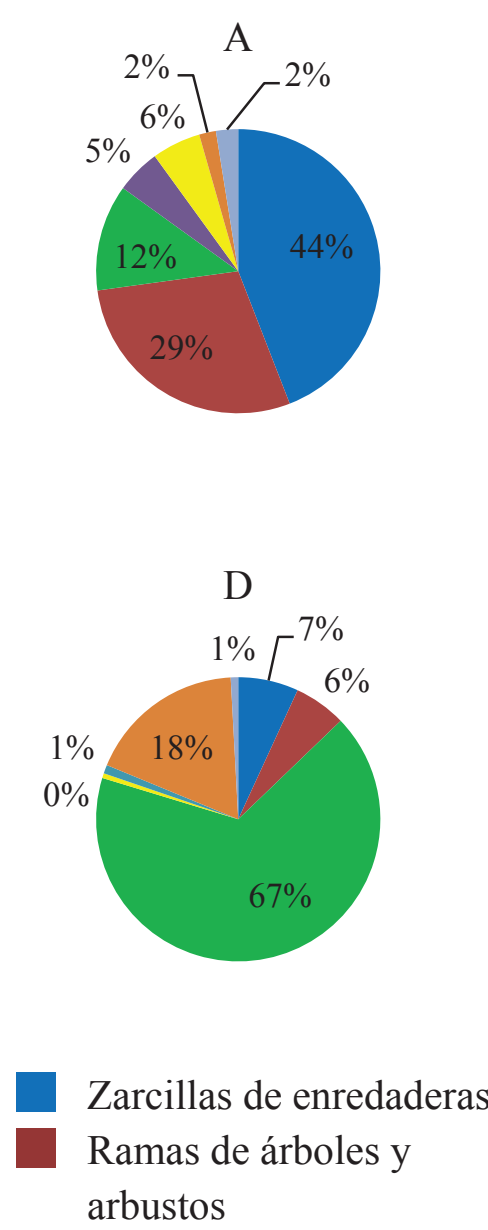
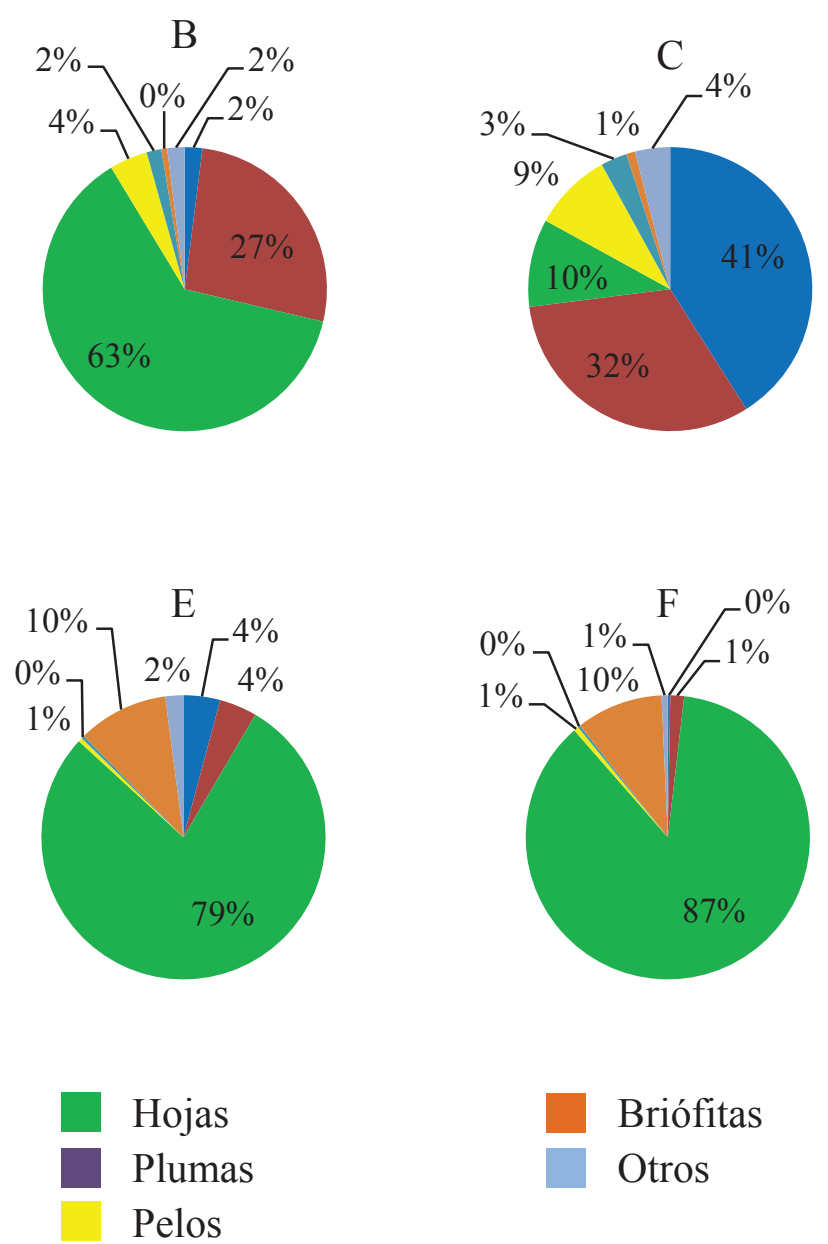

\section{Briófitas \\ Otros}

Figura 1. Frecuencia de ocurrencia de los ítems utilizados en la construcción de nidos de vertebrados nidificadores de cavidades en el bosque templado andino del sur de Chile (2010-2013). Aphrastura spinicauda (A), Troglodytes aedon (B), Tachycineta meyeni (C), Dromiciops gliroides (D), Rattus rattus (E), Irenomys tarsalis/Oligoryzomys longicaudatus (F).

Frequency of occurrence of items used in nests by cavity-nesting vertebrates in the Andean temperate forest of southern Chile (2010-2013). Aphrastura spinicauda (A), Troglodytes aedon (B), Tachycineta meyeni (C), Dromiciops gliroides (D), Rattus rattus (E), Irenomys tarsalis/Oligoryzomys longicaudatus $(\mathrm{F})$. 
A

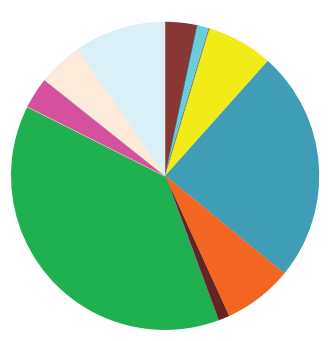

D

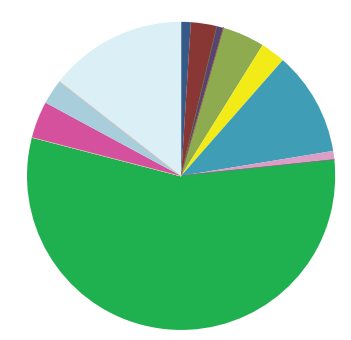

B

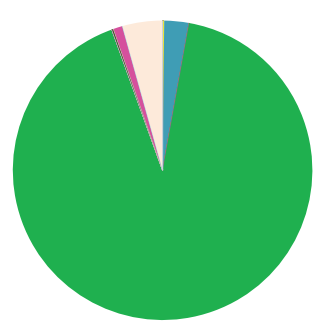

E

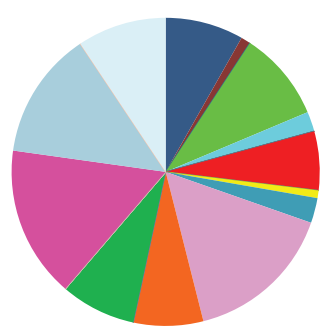

C

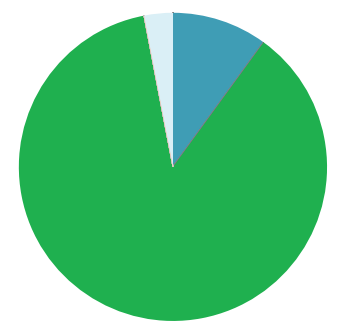

F

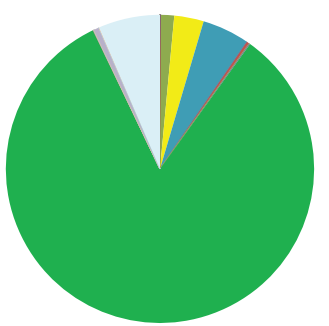

Aextoxicon punctatum

Aristotelia chilensis

Dasyphyllum diacantoides

Eucryphia cordifolia

Gevuina avellana

Lomatia hirsuta

Lophozonia alpina

Nothofagus dombeyi

Lophozonia obliqua

$\square$ Persea lingue
Pinus radiata

Saxegothaea conspicua

Chusquea spp.

Myrceugenia planipes

Tillandsia usneoides

Boquila trifololiata

Lapageria rosea

Poacea spp.

Desconocido

Figura 2. Proporción de hojas presentes en los nidos de Aphrastura spinicauda (A), Troglodytes aedon (B), Tachycineta meyeni (C), Dromiciops gliroides (D), Rattus rattus (E), e Irenomys tarsalis/Oligoryzomys longicaudatus (F) en el bosque templado andino del sur de Chile (2010-2013).

Proportion of leaves in nests of Aphrastura spinicauda (A), Troglodytes aedon (B), Tachycineta meyeni (C), Dromiciops gliroides (D), Rattus rattus (E) and Irenomys tarsalis/Oligoryzomys longicaudatus (F) in the Andean temperate forest of southern Chile (2010-2013).

nidos dependiendo del hábitat. Por ejemplo, tanto Moreno et al. (2005) como Celis-Diez et al. (2012), en la Isla grande de Chiloé detallan la presencia de helechos de la familia Hymenophyllaceae en la composición de nidos de A. spinicauda y D. gliroides, respectivamente. Lo anterior coincide con que la familia de helechos epífitos Hymenophyllaceae es la más abundante en la isla (Veblen y Schlegel 1982). Además, los materiales utilizados por $A$. spinicauda en la construcción de sus nidos en Chiloé coinciden en términos generales con las especies de plantas disponibles en el ambiente, destacando los tallos finos de epífitas, helechos y rizomas (Veblen y Schlegel 1982). Por otro lado, estudios realizados por Quilodrán et al. (2012) y Muñoz-Pedreros et al. (1996), ambos en plantaciones de $P$. radiata, detallan la alta presencia de acículas de pino en la construcción de nidos de A. spinicauda y T. aedon, respectivamente.

Entre aves y mamíferos se observó una diferencia en la utilización de materiales para nidificar. Las aves fueron más generalistas en cuanto al uso de materiales, mientras que los mamíferos utilizaron mayormente hojas y briófitas. Estas últimas podrían tener un rol biocida contra patógenos, otorgando mayor protección a las crías. Dentro de las hojas, los mamíferos utilizaron principalmente hojas de Chusquea spp., a excepción de la especie exótica R. rattus que utilizó en proporciones similares hojas de Boquila trifoliolata, Persea lingue (R. et Pav.) Nees (lingue) y Lapageria rosea. Esto coincide, en términos de selección de materiales, con las descripciones previas de nidos de D. gliroides en la zona continental, en donde la mayoría de 
los nidos fueron construidos principalmente con Chuquea spp. (Jiménez y Rageot 1979). A pesar de la diferencia en la proporción de materiales para la construcción del nido en las aves, todas las especies se asemejaron en buscar materiales aislantes y térmicos (i.e. plumas y hojas) para el revestimiento de su taza interna (Hilton et al. 2004).

Este estudio aporta con un nuevo rol de Chusquea spp. en la ecología de especies que habitan el bosque templado de Sudamérica: su uso frecuente en los nidos de algunos vertebrados nidificadores de cavidades. Los nidos de $D$. gliroides, I. tarsalis y $O$. longicaudatus fueron fuertemente reforzados con un tejido enmarañado de Chusquea spp., probablemente asociado a mantener una temperatura adecuada en los nidos (Hilton et al. 2004, Mainwaring et al. 2014). Lo anterior podría deberse al carácter estable de las hojas como material aislante (Reid et al. 2002), pudiendo ser el mejor aislante en condiciones de alta humedad y precipitación (no así las plumas), como son las condiciones de invierno en que estas especies hibernan y/o se refugian. Por otra parte, ha sido reportado que los tejidos de Chusquea spp. tienen un $\mathrm{pH}$ de cinco o menor (Rijo et al. 1987). El pH ácido produce una drástica reducción de la supervivencia de los microorganismos; por lo tanto, el uso de Chusquea spp. podría evitar la proliferación de patógenos (Dubiec et al. 2013). De esa forma, el uso de hojas de las especies del género Chusquea en los nidos podría inhibir la proliferación de patógenos, aunque también podría aportar en la termorregulación interna, reducción de la depredación (aportando volumen al nido; Hansell 2000) e, incluso, podría jugar un rol en la selección sexual (Mainwaring et al. 2014).

A pesar de la aparente generalización en el uso de recursos en los nidos por los vertebrados nidificadores de cavidades en el bosque templado, el presente estudio demuestra que $A$. spinicauda y $D$. gliroides prefieren algunos recursos presentes en el hábitat reproductivo y/o de hibernación por sobre otros. Aphrastura spinicauda construyó sus nidos mayoritariamente en base a trepadoras, material disponible en el $100 \%$ de las parcelas donde se ubicaron las cajas-nido. Este resultado coincide con la abundancia de trepadoras en bosques de la zona estudiada (Veblen y Schlegel 1982, Altamirano 2014). En la zona andina, A. spinicauda mostró preferencia por acículas de $P$. radiata al utilizar este material en el $20 \%$ de los nidos a pesar de no ser abundante en el sitio de estudio $(6,5 \%$ de las parcelas). Lo anterior puede deberse a que este material permite entretejer la base del nido, pudiendo suplir el rol de las trepadoras. De hecho, el promedio de trepadoras en los nidos sin acículas de $P$. radiata fue mayor que el promedio encontrado en los nidos con presencia de acículas de esta especie, disminuyendo de $44,1 \%$ a $31,0 \%$ respectivamente. Respecto al ítem hojas, $A$. spinicauda mostró preferencia por Chusquea spp. y rechazo de L. obliqua. Esta preferencia de Chusquea spp. podría deberse a la flexibilidad de ésta para poder entretejer este material dentro del nido, al contrario de la hoja de L. obliqua que, por su pequeño tamaño ( $5 \mathrm{~cm}$ de largo, aproximadamente) y poca flexibilidad, no permite el entretejido.

Por su parte, $D$. gliroides mostró una preferencia por hojas de Chusquea spp. y un rechazo por trepadoras en la construcción de sus nidos. Lo anterior podría deberse a que este mamífero no necesita entretejer sus nidos con una estructura rígida para sostener una estructura firme o taza interna. El largo de las hojas de Chusquea spp. permite un entretejido flexible que mantiene un espacio interno para la reproducción e hibernación. Finalmente, D. gliroides utilizó hojas de L. obliqua solo cuando estas se encontraban presentes dentro del hábitat reproductivo. Esto se puede deber a que $D$. gliroides sólo utilizaría estas hojas para otorgar volumen a su nido, completando el interior de la cavidad y protegiendo aún más el espacio interno donde se localizarían los adultos y/o las crías.

\section{CONCLUSIONES}

Los estudios de naturaleza descriptiva aportan al conocimiento de la historia natural, por ejemplo, en la composición de materiales utilizados en los nidos fabricados para la reproducción, hibernación y/o descanso de las especies. Sin embargo, al evaluar la preferencia que tienen las especies sobre los recursos disponibles, se puede dilucidar que la utilización de ciertos materiales podría no corresponder con la disponibilidad de materiales en sus hábitats. Este trabajo evidencia la amplia variedad de materiales utilizados en la construcción de nidos por siete especies nidificadoras de cavidades del bosque templado. Por otra parte, las hojas de Chusquea spp. son preferidas por dos vertebrados nidificadores de cavidades. Chusquea spp. podría influenciar el éxito reproductivo de estas especies, proporcionando protección contra depredadores (debido a la dificultad de capturar a la presa en el interior de la cavidad) y/o teniendo un efecto biocida contra patógenos. Estos posibles mecanismos asociados al uso de Chusquea spp. aún deben ser evaluados.

\section{AGRADECIMIENTOS}

Esta investigación fue financiada por Fondos de Protección Ambiental (09-078-2010 y 9-I-009-12) del Ministerio del Medio Ambiente, The Peregrine Fund, The Rufford Small Grants for Nature Conservation (14397-2), Francois Vuilleumier Fund for Research on Neotropical Birds (Sociedad de Ornitología Neotropical), e Idea Wild. Este estudio fue posible gracias al invaluable apoyo de Alberto Dittborn, Jerry Laker, Cristina Délano, Mónica Sabugal, Ricardo Timmerman y la Comunidad de Kawellucó quienes permitieron el desarrollo de esta investigación en sus predios. Las sugerencias y comentarios del editor y dos revisores anónimos ayudaron a mejorar considerablemente el manuscrito. T. A. Altamirano, J. T. Ibarra, M. De la Maza fueron becarios de postgrado de la Comisión Nacional de Investigación Científica y Tecnológica de Chile (CONICYT). 


\section{REFERENCIAS}

Altamirano TA. 2014. Breeding ecology of cavity-nesting birds in the Andean temperate forest of southern Chile. Tesis Doctorado en Ciencias. Santiago, Chile. Departamento de Ecosistemas y Medio Ambiente, Facultad de Agronomía e Ingeniería Forestal, Pontificia Universidad Católica de Chile. $145 \mathrm{p}$.

Altamirano TA, JT Ibarra, M de la Maza, SA Navarrete, C Bonacic. 2015. Reproductive life-history variation in a secondary cavity-nester across an elevational gradient in Andean temperate ecosystems. The Auk: Ornithological Advances 132: 826-835.

Álvarez E, E Barba. 2009. ¿Cómo afecta la calidad del nido per se al proceso de incubación? Una aproximación experimental. Revista Catalana d'Ornitologia 25: 11-18.

Atienzar F, E Belda, J Greño. 2010. Comparación de materiales utilizados en la construcción del nido y de los parámetros reproductores en el chochín Troglodytes troglodytes en la Font Roja y en la Sierra de Mariola. Iberis 8: 17-22.

Celis-Diez JL, J Hetz, PA Marín Vial, G Fuster, P Necochea, RA Vásquez, FM Jaksic, JJ Armesto. 2012. Population abundance, natural history, and habitat use by the arboreal marsupial Dromiciops gliroides in rural Chiloé Island, Chile. Journal of Mammalogy 93: 134-148.

Clark L, JR Mason. 1985. Use of nest material as insecticidial and anti-pathogenic agents by the European Starling. Oecología 67: 169-176.

Collias NE, EC Collias. 1984. Nest building and bird behaviour. Princeton, USA. Princeton University Press.

Dubiec A, I Gózdz, TD Mazgagski. 2013. Green plant material in avian nests. Avian Biology Research 6: 133-146.

Hansell M. 2000. Bird nests and construction behaviour. Cambridge, UK. Cambridge University Press. 295 p.

Hilton GM, MH Hansell, GD Ruxton, JM Reid, P Monaghan. 2004. Using artificial nests to test importance of nesting material and nest shelter for incubation energetics. The Auk 121: 777-787.

Ibarra JT, K Martin. 2015. Biotic homogenization: loss of avian functional richness and habitat specialists in disturbed Andean temperate forests. Biological Conservation 192: 418427.

Ippi S, R Vásquez, J Moreno, S Merino, CP Villavicencio. 2012. Breeding biology of the Southern House Wren on Chiloé Island, southern Chile. The Wilson Journal of Ornithology
124: 531-537.

Jiménez J, R Rageot. 1979. Notas sobre la biología del monito del monte (Dromiciops australis Philippi 1893). Anales del Museo de Historia Natural 12: 83-88.

Hall LS, PR Krausman, ML Morrison. 1997. The habitat concept and a plea for standard terminology. Wildlife Society Bulletin 25: 173-182.

Liljesthröm M, A Schiavini, JC Reboreda. 2009. Chilean swallows (Tachycineta meyeni) adjust the number of feathers added to the nest with time of breeding. The Wilson Journal of Ornithology 121: 783-788.

Mainwaring MC, IR Hartley, MM Lambrechts, DC Deeming. 2014. The design and function of bird's nests. Ecology and Evolution 4: 3909-3928.

Martin K, J Eadie. 1999. Nest webs: a community-wide approach to the management and conservation of cavity-nesting forest birds. Forest Ecology and Management 115: 243-257.

Moreno J, S Merino, R Vásquez, JJ Armesto. 2005. Breeding biology of the thorn-tailed rayadito (Furnariidae) in southtemperate rainforests of Chile. The Condor 107: 69-77.

Muñoz-Pedreros A, A Gantz, M Saavedra. 1996. Nidos artificiales en plantaciones de Pinus radiata en el sur de Chile: ¿Una herramienta para mitigar impactos ambientales negativos? Revista Chilena de Historia Natural 69: 393-400.

Quilodrán C, R Vásquez, C Estades. 2012. Nesting of the ThornTailed Rayadito (Aphrastura spinicauda) in a Pine Plantation in Southcentral Chile. The Wilson Journal of Ornitho$\log$ 124: 737-742.

Reid JM, W Cresswell, S Holt, RJ Mellanby, DP Whitfield, GD Ruxton. 2002. Nest scrape design and clutch heat loss in Pectoral Sandpipers (Calidris melanotos). Functional Ecology 16: 305-312.

Rijo C, H Poblete, JE Díaz-Vaz, M Torres, A Fernández. 1987. Estudios de algunas características anatómicas, físicas y químicas de Chusquea culeou (Coligue). Bosque 8(1): 59-61.

Rojas I, P Becerra, N Gálvez, J Laker, C Bonacic, A Hester. 2011. Relationship between fragmentation, degradation and native and exotic species richness in an Andean temperate forest of Chile. Gayana Botánica 68: 163-175.

Veblen TT, FM Schlegel. 1982. Reseña ecológica de los bosques del sur de Chile. Bosque 4(2): 73-115.

Vuilleumier F. 1985. Forest birds of Patagonia: ecological geography, speciation, endemism, and faunal history. Ornithological Monographs 36: 255-304. 